\title{
THE INFIMAL VALUE FUNCTIONAL AND THE UNIFORMIZATION OF HIT-AND-MISS HYPERSPACE TOPOLOGIES
}

\author{
GERALD BEER AND ROBERT TAMAKI \\ (Communicated by James E. West) \\ Dedicated to the memory of Wim Vervaat
}

\begin{abstract}
We give necessary and sufficient conditions for the uniformizability of hit-and-miss and proximal hit-and-miss hyperspace topologies defined on the nonempty closed subsets $\mathrm{CL}(X)$ of a Hausdorff uniform space $\langle X, \mathscr{U}\rangle$. In the case of uniformizability, one can always find a family $F$ of continuous functions on $X$ into $[0,1]$ so that the hyperspace topology is the weak topology induced by $\left\{m_{f}: f \in \mathscr{F}\right\}$, where for each $f, m_{f}: \operatorname{CL}(X) \rightarrow[0,1]$ is the infimal value functional defined by $m_{f}(A)=\inf \{f(x): x \in A\}$.
\end{abstract}

\section{INTRODUCTION}

Let $X$ be a Hausdorff uniform space, and let $\mathrm{CL}(X)$ be the nonempty closed subsets of $X$. A fundamental class of topologies on $\mathrm{CL}(X)$ are the hit-andmiss hyperspace topologies, having the Vietoris topology and the Fell topologies as prototypes. To describe these topologies, for each $A \subset X$, define subsets $A^{+}$ and $A^{-}$of $\mathrm{CL}(X)$ as follows:

$$
\begin{aligned}
& A^{+} \equiv\{B \in \mathrm{CL}(X): B \subset A\}, \\
& A^{-} \equiv\{B \in \mathrm{CL}(X): B \cap A \neq \varnothing\} .
\end{aligned}
$$

The Vietoris topology $\tau_{V}[\mathrm{Mi}, \mathrm{KT}]$ on $\mathrm{CL}(X)$ has as a subbase all sets of the form $V^{-}$where $V$ is an arbitrary open subset of $X$ plus all sets of the form $W^{+}$where $W$ is an arbitrary open subset of $X$. The weaker Fell topology $\tau_{F}$ [Fe, $\mathrm{Po} 2, \mathrm{At}, \mathrm{KT}]$ on $\mathrm{CL}(X)$ has as a subbase all sets of the form $V^{-}$where $V$ is an arbitrary open subset of $X$ plus all sets of the form $W^{+}$where $W$ is an arbitrary open subset of $X$ with compact complement. In each case, there are two types of subbasic open sets: those that "hit" a variable open set plus those that "miss" a closed set that belongs to a prescribed family of closed sets. This motivates the following definition.

Received by the editors January 11, 1993.

1991 Mathematics Subject Classification. Primary 54B20.

Key words and phrases. Hyperspace topology, hit-and-miss topology, uniformity, weak topology, infimal value functional, Vietoris topology, Fell topology. 
1.1. Definition. Let $\langle X, \tau\rangle$ be a Hausdorff space, and let $\Delta$ be a subfamily of $\mathrm{CL}(X)$ containing the singletons. The hit-and-miss hyperspace topology $\tau_{\Delta}^{+}$on $\mathrm{CL}(X)$ has as a subbase all sets of the form $V^{-}$where $V$ is an arbitrary open subset of $X$ plus all sets of the form $\left(D^{c}\right)^{+}$where $D \in \Delta$.

Now suppose $\langle X, \mathscr{U}\rangle$ is a uniform space. If $U$ is an entourage and $A \subset X$, we write $U[A]$ for $\{x \in X: \exists a \in A$ with $(x, a) \in U\}$. Given subsets $A$ and $B$ of $X$ we write $A \subset \subset B$ if $U[A] \subset B$ for some $U \in \mathscr{U}$ and $B^{++}$for $\{A \in \mathrm{CL}(X): A \subset \subset B\}$. We say that (disjoint) subsets $A$ and $B$ of $X$ are far provided there exists $U \in \mathscr{U}$ such that $U[A] \cap U[B]=\varnothing$. Clearly, $A$ is far from $B$ if and only if $A \in\left(B^{c}\right)^{++}$. Farness so defined is not really a uniform notion but a proximity notion [NW], in that different uniformities can give rise to the same farness relation. We may replace miss by far from in Definition 1.1 to obtain another class of hyperspace topologies, as follows (see, e.g., [BLLN, BL, Be1, Be2, DCNS, SZ, BT]):

1.2. Definition. Let $\langle X, \mathscr{U}\rangle$ be a Hausdorff uniform space, and let $\Delta$ be a subfamily of $\mathrm{CL}(X)$ containing the singletons. The proximal hit-and-miss hyperspace topology $\tau_{\Delta}^{++}$on $\mathrm{CL}(X)$ has as a subbase all sets of the form $V^{-}$ where $V$ is an arbitrary open subset of $X$ plus all sets of the form $\left(D^{c}\right)^{++}$ where $D \in \Delta$.

Clearly if $K$ is compact, then $\left(K^{c}\right)^{+}=\left(K^{c}\right)^{++}$and so the Fell topology agrees with the proximal Fell topology, independent of the choice of the underlying uniformity. In general $\tau_{\Delta}^{++}$and $\tau_{\Delta}^{+}$are noncomparable; the precise relationship between $\tau_{\Delta}^{++}$and $\tau_{\Delta}^{+}$in the metrizable case is spelled out in [BT].

It has now become evident that for many applications, proximal hit-and-miss hyperspace topologies overshadow their hit-and-miss counterparts. Convincing evidence for this sweeping claim can be produced in the context of normed spaces. When $\Delta=$ the closed balls, then $\tau_{\Delta}^{++}$-convergence of a net $\left\langle A_{\lambda}\right\rangle$ of closed sets to $A$ corresponds to the Wijsman convergence [Wi, FLL] of the net, i.e., the convergence for each $x \in X$ of $\left\langle d\left(x, A_{\lambda}\right)\right\rangle$ to $d(x, A)$ [SZ]. When $\Delta=$ the closed and bounded convex sets, then strong convergence of continuous linear functionals corresponds to the $\tau_{\Delta}^{++}$-convergence of their graphs and to the convergence of level sets at fixed heights [BB]. The restriction of $\tau_{\Delta}^{++}$to the closed and bounded convex sets yields the fundamental topology of Joly [Jo], which has a number of elegant characterizations in terms of convex duality [ $\mathrm{Be} 2$, $\mathrm{AB}, \mathrm{Be} 3]$. If $\Delta$ is still the larger class of all convex sets, then $\tau_{\Delta}^{++}$-convergence of closed convex sets corresponds to pointwise convergence of distance functionals plus pointwise convergence of support functionals for the sets, each viewed as functionals of a set variable with the point argument held fixed [He, Be1].

It is known that the Vietoris topology is uniformizable if and only if $X$ is normal [Mi]; indeed, if $X$ is given the uniformity of the Stone-Cech compactification, then the induced Hausdorff uniformity [NW, $\S 15]$ on $\mathrm{CL}(X)$ is compatible with the Vietoris topology. If $X$ is locally compact, then $\left\langle\mathrm{CL}(X), \tau_{F}\right\rangle$ can be embedded as a dense open subset in a compact Hausdorff space (see, e.g., [At, KT]) and thus is locally compact Hausdorff. Without local compactness, the Fell topology is neither Hausdorff nor regular [Po2]. Thus uniformizability of $\left\langle\mathrm{CL}(X), \tau_{F}\right\rangle$ is equivalent to local compactness of $X$.

The aim of this paper is to settle the following general question: when is a hit-and-miss or a proximal hit-and-miss topology uniformizable? Uniformiz- 
ability is equivalent to regularity but not to the Hausdorff separation property. Our answer to this question yields attractive characterizations of convergence in the Vietoris and Fell topologies in terms of infimal value functionals when these topologies are uniformizable. Finally, we shall show that a hit-and-miss topology cannot be uniformizable unless it is a proximal hit-and-miss hyperspace topology in the first place!

\section{Preliminaries}

In the sequel, $\langle X, \mathscr{U}\rangle$ will be a Hausdorff uniform space and $\mathrm{CL}(X)$ will denote the nonempty closed subsets of $X$. We write $C(X, \mathbf{R})$ (resp. $C(X, \mathbf{I})$ ) for the continuous functions from $X$ to $\mathbf{R}$ (resp. $[0,1]$ ), and $U C(X, \mathbf{R})$ (resp. $U C(X, \mathbf{I})$ ) for the uniformly continuous functions from $X$ to $\mathbf{R}$ (resp. $[0,1])$. If $f \in C(X, \mathbf{R})$ and $A \in \operatorname{CL}(X)$, then $m_{f}(A) \equiv \inf \{f(x): x \in A\}$ is called the infimal value of $f$ on $A$. If $f \in C(X, \mathbf{R})$ and $\alpha \in \mathbf{R}$, we write $\operatorname{slv}(f ; \alpha)$ for the sublevel set of $f$ at height $\alpha: \operatorname{slv}(f ; \alpha) \equiv\{x \in X: f(x) \leq \alpha\}$. Each sublevel set is closed, but some may be empty.

We call a topology on $\mathrm{CL}(X)$ a hyperspace topology. A hyperspace topology $\sigma$ is called admissible [Mi] provided the assignment $x \rightarrow\{x\}$ is a topological embedding of $X$ into $\langle\mathrm{CL}(X), \sigma\rangle$. We collect some simple known facts about hit-and-miss and proximal hit-and-miss topologies in a lemma, whose proof is left to the reader.

2.1. Lemma. Let $\langle X, \mathscr{U}\rangle$ be a Hausdorff uniform space, let $\Delta$ be a family of nonempty closed sets containing that singletons, and let $\sigma$ denote either $\tau_{\Delta}^{+}$or $\tau_{\Delta}^{++}$. Then $\sigma$ satisfies the $T_{1}$ separation axiom and is admissible. Furthermore, if $\left\langle A_{\lambda}\right\rangle_{\lambda \in \Lambda}$ is an increasing net of sets in $\mathrm{CL}(X) \quad\left(\lambda_{1} \leq \lambda_{2} \Rightarrow A_{\lambda_{1}} \subset A_{\lambda_{2}}\right)$, then $\left\langle A_{\lambda}\right\rangle_{\lambda \in \Lambda}$ is $\sigma$-convergent to $\operatorname{cl}\left(\bigcup_{\lambda \in \Lambda} A_{\lambda}\right)$.

If $\Delta$ is a class of nonempty closed subsets of $X$, we write $\Sigma(\Delta)$ for the set of finite unions of elements of $\Delta$. It is clear that a base for the hit-and-miss topology $\tau_{\Delta}^{+}$consists of all sets of the form $\left(S^{c}\right)^{+} \cap V_{1}^{-} \cap V_{2}^{-} \cap \cdots \cap V_{n}^{-}$where $S \in \Sigma(\Delta)$ and $\left\{V_{1}, V_{2}, \ldots, V_{n}\right\}$ is a finite collection of open subsets, whereas a base for $\tau_{\Delta}^{++}$consists of all sets of the form $\left(S^{c}\right)^{++} \cap V_{1}^{-} \cap V_{2}^{-} \cap \cdots \cap V_{n}^{-}$ where $S \in \Sigma(\Delta)$ and $\left\{V_{1}, V_{2}, \ldots, V_{n}\right\}$ are open.

The following property for $\Delta$ was considered by Poppe [Po2] and is implicit in [Ve].

2.2. Definition. Let $\Delta$ be a family of nonempty closed subsets of a Hausdorff space $X$ containing the singletons. We call $\Delta$ a Urysohn family provided whenever $D \in \Delta$ and $A \in \mathrm{CL}(X)$ are disjoint there exists $S \in \Sigma(\Delta)$ such that $D \subset \operatorname{int} S \subset S \subset A^{c}$.

One of the key ideas in this paper is a natural extension of Definition 2.2 to uniform spaces as follows.

2.3. Definition. Let $\langle X, \mathscr{U}\rangle$ be a Hausdorff uniform space, and let $\Delta$ be a family of nonempty closed subsets of $X$ containing the singletons. We call $\Delta$ a uniformly Urysohn family provided whenever $D \in \Delta$ and $A \in \mathrm{CL}(X)$ are far there exist $S \in \Sigma(\Delta)$ and $U \in \mathscr{U}$ such that $U[D] \subset S \subset A^{c}$.

Definition 2.3 is an extension of Definition 2.2 rather than just an analogue, in that each Urysohn family is a uniformly Urysohn family in disguise, as we 
now explain. First, some background material is in order.

Let $F$ be a family of real functionals defined on a set $E$. The weak topology induced by $\mathscr{F}$ on $E$ is the weakest topology on $E$ making each function in $\mathscr{F}$ continuous. The uniformity $\mathscr{U}(\mathscr{F})$ on $E$ determined by $\mathscr{F}$ has as a base for its entourages all sets of the form

$$
\left\{\left(e_{1}, e_{2}\right): \forall f \in \widehat{\mathscr{F}},\left|f\left(e_{1}\right)-f\left(e_{2}\right)\right|<\varepsilon\right\}
$$

where $\varepsilon>0$ and $\widehat{\mathscr{F}}$ is a finite subset of $\mathscr{F}$. This not only is compatible with the weak topology but also makes each element of $\mathscr{F}$ uniformly continuous. Now let $\Delta$ be an arbitrary Urysohn family. If $D \in \Delta$ and $A \in \mathrm{CL}(X)$ are disjoint, then a modification of the standard proof of Urysohn's Lemma for the functional separation of disjoint closed sets in a normal space gives $f \in C(X, \mathbf{I})$ with $f(D)=0$ and $f(A)=1$ (see the proof of Lemma 3.1 below). Since $\Delta$ contains the singletons, $C(X, \mathbf{R})$ separates points from closed sets, and so $X$ is already completely regular. Not only is $\mathscr{U}(C(X, \mathbf{R}))$ compatible with the underlying topology, but also the Urysohn family $\Delta$ becomes uniformly Urysohn with respect to $\mathscr{U}(C(X, \mathbf{R}))$. As a particular example, in a locally compact Hausdorff space, the Urysohn family of compact sets is uniformly Urysohn with respect to $\mathscr{U}(C(X, \mathbf{R}))$.

On the other hand, a Urysohn family need not be uniformly Urysohn with respect to the given uniformity $\mathscr{U}$ for the space, even when $\mathscr{U}$ is a metric uniformity. To see this, we consider the following metric subspace $X$ of $l_{\infty}$ introduced in Example 3.1 of [BT]:

$$
X=\{\theta\} \cup\left\{e_{2 n}: n \in \mathbf{Z}^{+}\right\} \cup\left\{\frac{n+1}{n} e_{n}: n \in \mathbf{Z}^{+}\right\},
$$

where $\theta$ is the origin of the space and $\left\{e_{n}: n \in \mathbf{Z}^{+}\right\}$is the standard set of unit vectors. For our distinguished family of closed sets $\Delta$, we take the closed balls. Evidently, the space is discrete, in which case each family of closed sets is Urysohn. But with $A=\left\{\frac{n+1}{n} e_{n}: n\right.$ odd $\}$ and $D=$ the closed ball with radius 1 and center $\theta$, we have $A$ far from $D$, yet the uniformly Urysohn condition fails for this pair. It is easy to see that a uniformly Urysohn family need not be Urysohn: $\Delta=\mathrm{CL}(X)$ in any complete regular nonnormal space does the job.

The following inclusion is noteworthy.

2.4. Lemma. Let $\langle X, \mathscr{U}\rangle$ be a Hausdorff uniform space, and let $\Delta \subset \mathrm{CL}(X)$ be a uniformly Urysohn family. Then $\tau_{\Delta}^{++} \subset \tau_{\Delta}^{+}$on $\mathrm{CL}(X)$.

Proof. It suffices to show that if $D \in \Delta$, then $\left(D^{c}\right)^{++}$contains a $\tau_{\Delta}^{+}$-neighborhood of any $A \in\left(D^{c}\right)^{++}$. Since $A$ is far from $D$, by the definition of uniformly Urysohn, we can find a $V \in \mathscr{U}$ and $S \in \Sigma(\Delta)$ such that

$$
D \subset V[D] \subset S \subset A^{c} .
$$

It is now easy to see that $A \in\left(S^{c}\right)^{+} \subset\left(D^{c}\right)^{++}$.

The uniform Urysohn property does not characterize the above inclusion, for in any Hausdorff space we have equality of $\tau_{\Delta}^{+}$and $\tau_{\Delta}^{++}$for $\Delta=$ the compact sets. For a precise characterization of this inclusion in the case of a metric uniformity, see Theorem 3.2 of [BT]. 
As shown by Poppe [Po2], the Urysohn property for $\Delta$ characterizes regularity for $\tau_{\Delta}^{+}$, and we shall see that the uniformly Urysohn property for $\Delta$ characterizes regularity for $\tau_{\Delta}^{++}$. On the other hand, the same property for $\Delta$ characterizes the Hausdorff separation property for either hyperspace.

2.5. Lemma. Let $\langle X, \mathscr{U}\rangle$ be a Hausdorff uniform space, and let $\Delta$ be a subfamily of $\mathrm{CL}(X)$. The following are equivalent:

(1) $\tau_{\Delta}^{+}$is Hausdorff;

(2) $\tau_{\Delta}^{++}$is Hausdorff;

(3) whenever $x \notin A \in \mathrm{CL}(X)$, there exists $S \in \Sigma(\Delta)$ such that $x \in$ int $S \subset$ $S \subset A^{c}$.

Proof. The equivalence of conditions (1) and (3) in the statement of Lemma 2.5 is again due to Poppe [Po1, Po2]. For (3) $\Rightarrow(2)$, suppose $\{A, B\} \subset \mathrm{CL}(X)$ and $a \in A-B$. Choose $V \in \mathscr{U}$ with $V[a] \cap V[B]=\varnothing$ and $S \in \Sigma(\Delta)$ such that $a \in$ int $S \subset S \subset V[a]$. Then $\left(S^{c}\right)^{++}$and (int $\left.S\right)^{-}$are disjoint neighborhoods of $B$ and $A$, respectively. For the proof of $(2) \Rightarrow(3)$, start with disjoint basic $\tau_{\Delta}^{++}$open sets $\mathscr{A}_{1}$ and $\mathscr{A}_{2}$ separating $A$ and $\{x\} \cup A$, say, $\mathscr{A}_{1} \equiv\left(S^{c}\right)^{+} \cap\left(\bigcap_{i=1}^{n} V_{i}^{-}\right)$ and $\mathscr{A}_{2} \equiv\left(T^{c}\right)^{+} \cap\left(\bigcap_{i=1}^{m} W_{i}^{-}\right)$, where $\{S, T\} \subset \Sigma(\Delta)$ and $V_{1}, V_{2}, \ldots, V_{n}$ are open subsets of $X$ disjoint from $S$ and $W_{1}, W_{2}, \ldots, W_{m}$ are open subsets of $X$ disjoint from $T$. Clearly, $A \cap S=\varnothing$, else $A \notin \mathscr{A}_{1}$; and for some $i \leq m$, $x \in W_{i}$, else $A \in \mathscr{A}_{2}$. Let the indices for which $x \in W_{i}$ be $\{1,2, \ldots, k\}$. Then $W_{i} \subset S$ for some $i \leq k$, else choosing $x_{i} \in W_{i} \cap S^{c}$ for each $i \leq m$, we get $A \cup\left\{x_{1}, x_{2}, \ldots, x_{k}\right\} \in \mathscr{A}_{1} \cap \mathscr{A}_{2}$, a contradiction.

Note that either the Urysohn property or the uniformly Urysohn property for a family $\Delta$ containing the singletons implies condition (3). This means that either hyperspace $\tau_{\Delta}^{+}$or $\tau_{\Delta}^{++}$can be Hausdorff without being regular.

\section{UNIFORMIZABILITY OF HIT-AND-MISS TOPOLOGIES}

Let $\langle X, \mathscr{U}\rangle$ be a uniform space, and let $\Delta$ be a uniformly Urysohn family of closed subsets of $X$. We aim to produce a subfamily $\mathscr{F}$ of $U C(X, \mathbf{I})$ such that $\tau_{\Delta}^{++}$is the weak topology determined by $\left\{m_{f}: f \in \mathscr{F}\right\}$. Such a program is not entirely original. In the context of metric spaces, using distance functionals for members of $\Delta$ as the family $\mathscr{F}$, we obtain $\tau_{\Delta}^{++}$as a weak topology provided $\Delta$ is "stable under enlargements" [BL, Theorem 2.1]. More general classes of uniformly continuous functions with prescribed sublevel set structure may be substituted for distance functionals, as observed by Sonntag and Zalinescu [SZ].

We will here work with classes of uniformly continuous functions whose sublevel sets are interposed by members of $\Sigma(\Delta)$ :

$$
\begin{array}{r}
\mathscr{F}_{\Delta, \mathbf{R}}^{++}=\{f \in U C(X, \mathbf{R}): \text { whenever inf } f<\alpha<\beta<\sup f, \exists S \in \Sigma(\Delta) \text { with } \\
\qquad \operatorname{slv}(f ; \alpha) \subset S \subset \operatorname{slv}(f ; \beta)\}, \\
\mathscr{F}_{\Delta, \mathbf{I}}^{++}=\{f \in U C(X, \mathbf{I}): \text { whenever inf } f<\alpha<\beta<\sup f, \exists S \in \Sigma(\Delta) \text { with } \\
\quad \operatorname{slv}(f ; \alpha) \subset S \subset \operatorname{slv}(f ; \beta)\} .
\end{array}
$$

The key to our analysis is a sharpening of Efremovic's proximal/uniform analogue of the Urysohn Lemma [Ef]. 
3.1. Lemma. Let $\langle X, \mathscr{U}\rangle$ be a Hausdorff uniform space, and let $\Delta$ be a family of closed subsets of $X$ containing the singletons. The following are equivalent:

(a) $\Delta$ is a uniformly Urysohn family.

(b) Whenever $S \in \Sigma(\Delta)$ and $A \in \mathrm{CL}(X)$ are far, there exist $S^{\prime} \in \Sigma(\Delta)$ and $U \in \mathscr{U}$ with $U[S] \subset S^{\prime}$ and $U\left[S^{\prime}\right] \subset A^{c}$.

(c) Whenever $D \in \Delta$ and $A \in \mathrm{CL}(X)$ are far, there exists $f \in \mathscr{F}_{\Delta, \mathrm{I}}^{++}$with $f[D]=0$ and $f[A]=1$.

Proof. (a) $\Rightarrow(\mathrm{b})$. Write $S=\bigcup_{i=1}^{n} D_{i}$ where $D_{i} \in \Delta$, and choose open $V_{i} \in \mathscr{U}$ such that $\left(V_{i} \circ V_{i}\right)\left[D_{i}\right] \subset A^{c}$. Choose $S_{i}^{\prime} \in \Sigma(\Delta)$ and entourages $U_{i} \subset V_{i}$ with $U_{i}\left[D_{i}\right] \subset S_{i}^{\prime} \subset V_{i}\left[D_{i}\right]$. Then $U \equiv \bigcap_{i=1}^{n} U_{i}$ and $S^{\prime} \equiv \bigcup_{i=1}^{n} S_{i}^{\prime}$ do the job.

(b) $\Rightarrow$ (c). Set $D=S_{0}$. Since $A$ and $S_{0}$ are far, by condition (b), there exist $U_{1} \in \mathscr{U}$ and $S_{1 / 2} \in \Sigma(\Delta)$ such that

$$
S_{0} \subset U_{1}\left(S_{0}\right) \subset S_{1 / 2} \subset U_{1}\left(S_{1 / 2}\right) \subset A^{c} .
$$

As a second step, since $S_{0}$ is far from $\left(S_{1 / 2}\right)^{c}$ and $S_{1 / 2}$ is far from $A$, there exist $U_{2} \in \mathscr{U}$ with $U_{2} \subset U_{1} \in \mathscr{U}$ and $S_{1 / 4}, S_{3 / 4} \in \Sigma(\Delta)$ such that

$$
S_{0} \subset U_{2}\left(S_{0}\right) \subset S_{1 / 4} \subset U_{2}\left(S_{1 / 4}\right) \subset S_{1 / 2} \subset U_{2}\left(S_{1 / 2}\right) \subset S_{3 / 4} \subset U_{2}\left(S_{3 / 4}\right) \subset A^{c} .
$$

Proceed inductively, using condition (b) repeatedly, until $S_{n / 2^{k}}$ and $U_{k}$ are constructed for all $n, k \in Z^{+}$such that $0 \leq n / 2^{k}<1$. The members of $\Sigma(\Delta)$ and the entourages we construct have the following properties:

(i) for $n=0,1,2, \ldots, 2^{k}-2$ we have $U_{k}\left(S_{n / 2^{k}}\right) \subset S_{(n+1) / 2^{k}}$;

(ii) the entourages selected get smaller as $k$ increases, i.e., $U_{k+1} \subset U_{k}$. Note that if $\alpha$ and $\beta$ are dyadic rationals in $(0,1)$ with $\alpha<\beta$, then $S_{\alpha} \subset \subset$ $S_{\beta}$. Now define $f: X \rightarrow \mathbf{I}$ as follows:

$$
f(x)= \begin{cases}\inf \left\{\alpha: x \in S_{\alpha}\right\} & \text { if } x \in \bigcup S_{\alpha}, \\ 1 & \text { otherwise. }\end{cases}
$$

Clearly $f(D)=0$ and $f(A)=1$. Furthermore, if $\alpha$ and $\beta$ are in $(0,1)$ with $\alpha<\beta$ and $\gamma$ is a dyadic rational strictly between them, then $\operatorname{slv}(f ; \alpha) \subset S_{\gamma} \subset$ $\operatorname{slv}(f ; \beta)$. To show that $f \in \mathscr{F}_{\Delta, \mathbf{I}}^{++}$, it remains to verify uniform continuity.

To this end, let $\varepsilon>0$ and choose $k \in \mathbf{Z}^{+}$large enough so that $2^{1-k}<\varepsilon$. We claim that if $(a, b) \in U_{k}$, then $|f(a)-f(b)|<\varepsilon$. Without loss of generality, suppose that $f(a)>f(b)$. We consider two cases. First, if $b \notin \bigcup\left\{S_{i / 2^{k}}: i<\right.$ $\left.2^{k}-1\right\}$, then

$$
f(b) \geq \frac{2^{k}-2}{2^{k}}=1-\frac{1}{2^{k-1}}>1-\varepsilon .
$$

Combined with $f(b)<f(a) \leq 1$, this yields the desired estimate. In the second case, there is a smallest $i<2^{k}-1$ such that $b \in S_{i / 2^{k}}$, because we are dealing with a finite number of sets ordered by inclusion. Since $(a, b) \in U_{k}$, it follows that $a \in S_{(i+1) / 2^{k}}$, so in particular $f(a) \leq \frac{i+1}{2^{k}}$. We compute

$$
|f(a)-f(b)|=f(a)-f(b) \leq \frac{i+1}{2^{k}}-\frac{i-1}{2^{k}}=\frac{1}{2^{k-1}}<\varepsilon,
$$

establishing uniform continuity of $f$. Thus, $f \in \mathscr{F}_{\Delta, \mathbf{I}}^{++}$.

(c) $\Rightarrow$ (a). Let $A \in \mathrm{CL}(X)$ and $D \in \Delta$ be far; by (c), we can choose $f \in$ $\mathscr{F}_{\Delta, \mathrm{I}}^{++}$with $f[D]=0$ and $f[A]=1$. By uniform continuity of $f$, there exists 
$U \in \mathscr{U}$ with $U[\operatorname{siv}(f ; 0)] \subset \operatorname{slv}(f ; 1 / 4)$. Choose $S \in \Sigma(\Delta)$ with $\operatorname{slv}(f ; 1 / 4) \subset$ $S \subset \operatorname{slv}(f ; 1 / 2)$. Then $U[D] \subset S \subset A^{c}$, as required.

3.2. Proposition. Let $\langle X, \mathscr{U}\rangle$ be a Hausdorff uniform space, and let $\Delta$ be a uniformly Urysohn family of closed subsets of $X$ containing the singletons. Let $A \in \mathrm{CL}(X)$, and let $\left\langle A_{\lambda}\right\rangle$ be a net in $\mathrm{CL}(X)$. The following are equivalent:

(1) $A=\tau_{\Delta}^{++}-\lim A_{\lambda}$;

(2) $\forall f \in \mathscr{F}_{\Delta, \mathbf{R}}^{++}$we have $\lim _{\lambda} m_{f}\left(A_{\lambda}\right)=m_{f}(A)$;

(3) $\forall f \in \mathscr{F}_{\Delta, \mathbf{I}}^{++}$we have $\lim _{\lambda} m_{f}\left(A_{\lambda}\right)=m_{f}(A)$.

Proof. (1) $\Rightarrow(2)$. Suppose $\left\langle A_{\lambda}\right\rangle \rightarrow A$ in $\tau_{\Delta}^{++}$, and let $f \in \mathscr{F}_{\Delta, \mathbf{R}}^{++}$. We must show that

$$
\lim _{\lambda} m_{f}\left(A_{\lambda}\right)=m_{f}(A) .
$$

First, suppose on the contrary that $\liminf _{\lambda} m_{f}\left(A_{\lambda}\right)<m_{f}(A)$. Choose scalars $\alpha<\beta$ strictly between $\liminf _{\lambda} m_{f}\left(A_{\lambda}\right)$ and $m_{f}(A)$. By the definition of $\mathscr{F}_{\Delta, \mathbf{R}}^{++}$, there exists $S \in \Sigma(\Delta)$ with $\operatorname{siv}(f ; \alpha) \subset S \subset \operatorname{slv}(f ; \beta)$. Since $\beta<m_{f}(A)$ and $f$ is uniformly continuous, $A$ is far from the $\operatorname{set} \operatorname{siv}(f ; \beta)$ and thus from $S$. Yet $\left\langle m_{f}\left(A_{\lambda}\right)\right\rangle$ is frequently below $\alpha$, and so $\left\langle A_{\lambda}\right\rangle$ frequently hits the set $S$, contradicting $\left\langle A_{\lambda}\right\rangle \rightarrow A$. Now suppose that $m_{f}(A)<\lim \sup _{\lambda} m_{f}\left(A_{\lambda}\right)$. Choosing $\eta$ strictly between $m_{f}(A)$ and $\lim \sup _{\lambda} m_{f}\left(A_{\lambda}\right)$, we note that $A$ hits the open set $f^{-1}(-\infty, \eta)$ but that $\left\langle A_{\lambda}\right\rangle$ frequently misses $f^{-1}(-\infty, \eta)$, which again contradicts $\left\langle A_{\lambda}\right\rangle \rightarrow A$.

(2) $\Rightarrow(3)$. This is immediate.

(3) $\Rightarrow(1)$. To see that $\left\langle A_{\lambda}\right\rangle \rightarrow A$ in $\tau_{\Delta}^{++}$, first suppose that for some $D \in \Delta$, we have $A \in\left(D^{c}\right)^{++}$, i.e., $A$ is far from $D$. By Lemma 3.1, we can construct an $f \in \mathscr{F}_{\Delta, \mathbf{I}}^{++}$such that $f(D)=0$ and $f(A)=1$ and, by $(3), \lim _{\lambda} m_{f}\left(A_{\lambda}\right)=1$. Since $f$ is uniformly continuous and $f(D)=0$, we can find $U \in \mathscr{U}$ such that $f(U[D]) \subset[0,1 / 2)$. Now $m_{f}(A)=1$ implies $m_{f}\left(A_{\lambda}\right)>1 / 2$ eventually. For all such $\lambda, U[D] \cap A_{\lambda}=\varnothing$, i.e., $A_{\lambda}$ is far from $D$.

Secondly, we need to show that if $A$ hits an open set $W$, then $\left\langle A_{\lambda}\right\rangle$ eventually hits $W$. Let $a$ be any point in $A \cap W$. Again use Lemma 3.1 to find a uniformly continuous function $f \in \mathscr{F}_{\Delta, \mathbf{I}}^{++}$such that $f(a)=0$ and $f\left(W^{c}\right)=1$. Since $a \in A$, we have $m_{f}(A)=0=\lim _{\lambda} m_{f}\left(A_{\lambda}\right)$. For the right side to be zero, $m_{f}\left(A_{\lambda}\right)$ would have to be eventually less than 1 , which can only happen if $A_{\lambda}$ eventually hits the open set $W$.

We now come to the main result of this paper.

3.3. Theorem. Let $\langle X, \mathscr{U}\rangle$ be a Hausdorff uniform space, and let $\Delta$ be a family of closed subsets of $X$ containing the singletons. Then $\tau_{\Delta}^{++}$is uniformizable if and only if $\Delta$ is uniformly Urysohn.

Proof. Suppose $\Delta$ is uniformly Urysohn. Proposition 3.2 tells us that the proximal hit-and-miss topology $\tau_{\Delta}^{++}$is the weak topology determined by $\mathscr{F}^{*} \equiv$ $\left\{m_{f}: f \in \mathscr{F}_{\Delta, \mathbf{I}}^{++}\right\}$. Thus, $\mathscr{U}\left(\mathscr{F}^{*}\right)$ is a compatible (totally bounded) uniformity for the topology.

For the converse, we show that regularity alone of $\tau_{\Delta}^{++}$ensures that $\Delta$ is uniformly Urysohn. This suffices, since uniformizability is equivalent to complete regularity. Let $A \in \mathrm{CL}(X)$ and $D \in \Delta$ be far. By regularity of the hyperspace, 
we can find a basic neighborhood $\mathscr{A}$ of $A$ in the topology $\tau_{\Delta}^{++}$such that

$$
A \in \mathscr{A} \subset \mathrm{cl} \mathscr{A} \subset\left(D^{c}\right)^{++} \text {. }
$$

We may write $\mathscr{A}=\left(S^{c}\right)^{++} \cap V_{1}^{-} \cap V_{2}^{-} \cap \cdots \cap V_{n}^{-}$where $S \in \Sigma(\Delta)$ and $\left\{V_{1}, V_{2}, \ldots, V_{n}\right\}$ are open subsets of $X$ each meeting $A$. Since $A \in \mathscr{A}$, the set $A$ is far from $S$. We claim that there exists $U \in \mathscr{U}$ such that $U[D] \subset S$. Suppose not. Choose an entourage $V_{0}$ such that $V_{0}[S] \cap A=\varnothing$, and let $\Omega$ be the family of open entourages contained in $V_{0}$, partially ordered by reverse inclusion. The assignment $V \rightarrow(V[S])^{c}$ on $\Omega$ is an increasing net in $\mathrm{CL}(X)$ which by Lemma 2.1 is convergent to $B \equiv \operatorname{cl}\left(\bigcup_{V \in \Omega}(V[S])^{c}\right)$. Since each set $(V[S])^{c}$ contains $A$ and is far from $S$, each belongs to $\mathscr{A}$. As we have chosen $\mathscr{A}$ so that cl $\mathscr{A} \subset\left(D^{c}\right)^{++}$, the set $B$ is far from $D$. On the other hand, if $W \in \mathscr{U}$ is arbitrary, then $W[D]$ meets $S^{c}$. Choosing $x \in W[D] \cap S^{c}$, there exists $V \in \Omega$ such that $(V \circ V)[x] \subset S^{c}$. Thus, $x \in W[D] \cap(V[S])^{c}$, and so $B$ is not far from $D$. This contradiction completes the proof.

The proof of Theorem 3.3 shows that regularity and complete regularity are equivalent for a proximal hit-and-miss topology. Our discussion of $\S 2$ shows that the Hausdorff separation property can hold for $\tau_{\Delta}^{++}$without these higher separation properties. We also remark for the record that when $\Delta=\operatorname{CL}(X)$, the hyperspace $\tau_{\Delta}^{++}$is always uniformizable. Special presentations of this topology as a weak topology are considered in some detail in [BLLN, BL] in the metric setting.

A natural way to extend the topology $\tau_{\Delta}^{++}$defined on $\mathrm{CL}(X)$ to $\mathrm{CL}(X) \cup\{\varnothing\}$ is to take as a local base of neighborhoods of the empty set all sets of the form $\left(S^{c}\right)^{++}$with $S \in \Sigma(\Delta)$.

3.4. Corollary. Let $\langle X, \mathscr{U}\rangle$ be a Hausdorff uniform space, and let $\Delta$ be a family cf closed subsets of $X$ containing the singletons. Then the extended proximal topology $\tau_{\Delta}^{++}$on $\mathrm{CL}(X) \cup\{\varnothing\}$ is uniformizable if and only if $\Delta$ is uniformly Urysohn.

Proof. Necessity is obvious. For sufficiency, we consider two cases: (i) $X \in$ $\Sigma(\Delta)$; (ii) $X \notin \Sigma(\Delta)$. In the first case, the empty set is an isolated point of the hyperspace, and we simply adjoin $(\varnothing, \varnothing)$ to each entourage in $\mathscr{U}\left(\mathscr{F}_{\Delta, \mathbf{I}}^{++}\right)$. In the second case, we extend each infimal value functional $m_{f}$ for $f \in \mathscr{F}_{\Delta, \mathbf{I}}^{++}$to a functional $\hat{m}_{f}: \operatorname{CL}(X) \cup\{\varnothing\} \rightarrow[0,1]$ in such a way that

$$
\left\langle A_{\lambda}\right\rangle \rightarrow \varnothing \quad \text { if and only if }\left\langle\hat{m}_{f}\left(A_{\lambda}\right)\right\rangle \rightarrow \hat{m}_{f}(\varnothing) \text {. }
$$

This would make the extended topology a weak topology and hence uniformizable. This is accomplished by setting $\hat{m}_{f}(\varnothing) \equiv \sup \{f(x): x \in X\}$.

We first verify that $\left\langle A_{\lambda}\right\rangle \rightarrow \varnothing$ implies $\left\langle\hat{m}_{f}\left(A_{\lambda}\right)\right\rangle \rightarrow \hat{m}_{f}(\varnothing)$ for $f \in \mathscr{F}_{\Delta, \mathbf{I}}^{++}$. If $f \equiv \alpha$, then $\hat{m}_{f} \equiv \alpha$, and there is nothing to show. Otherwise, let $\alpha$ be strictly between $m_{f}(X)$ and $\hat{m}_{f}(\varnothing)$. By the definition of $\mathscr{F}_{\Delta, \mathrm{I}}^{++}$, there exists $S \in \Sigma(\Delta)$ such that $\operatorname{slv}(f ; \alpha) \subset S$. Then $A_{\lambda} \in\left(S^{c}\right)^{++}$eventually implies $m_{f}\left(A_{\lambda}\right) \geq \alpha$ eventually; and since $\hat{m}_{f}\left(A_{\lambda}\right) \leq \hat{m}_{f}(\varnothing)$ for all $\lambda$, we have $\left\langle\hat{m}_{f}\left(A_{\lambda}\right)\right\rangle \rightarrow \hat{m}_{f}(\varnothing)$. Now suppose that $\forall f \in \mathscr{F}_{\Delta, \mathbf{I}}^{++},\left\langle\hat{m}_{f}\left(A_{\lambda}\right)\right\rangle \rightarrow \hat{m}_{f}(\varnothing)$. Fix $S \in \Sigma(\Delta)$; we may write $S=\bigcup_{i=1}^{n} D_{i}$ where $D_{i} \in \Delta$. Since we are assuming that $X \notin \Sigma(\Delta)$, we may choose for each $i$ a proper open subset $V_{i}$ of $X$ with $D_{i} \subset \subset V_{i}$ and 
$f_{i} \in \mathscr{F}_{\Delta, \mathrm{I}}^{++}$with $f_{i}\left(D_{i}\right)=0$ and $f_{i}\left(V_{i}^{c}\right)=1$. For each $i$ there exists an index $\lambda_{i}$ such that $m_{f_{i}}\left(A_{\lambda}\right)>1 / 2$ for $\lambda \geq \lambda_{i}$. Since each $f_{i}$ is uniformly continuous, $A_{\lambda}$ is far from $D_{i}$ when $\lambda \geq \lambda_{i}$. If we take an index $\lambda_{0} \geq \lambda_{i}$ for each $i$, then for each $\lambda \geq \lambda_{0}$ we have $A_{\lambda}$ far from $S$, as required.

As we have noted in $\S 2$, each Urysohn family $\Delta$ is uniformly Urysohn, provided that we equip the underlying space $X$ with the uniformity $\mathscr{U}(C(X, \mathbf{R}))$, and with this uniformity in mind, $\tau_{\Delta}^{+}$becomes $\tau_{\Delta}^{++}$. Moreover, if we define the function classes

$$
\begin{array}{r}
\mathscr{F}_{\Delta, \mathbf{R}}^{+}=\{f \in C(X, \mathbf{R}): \text { whenever inf } f<\alpha<\beta<\sup f, \exists S \in \Sigma(\Delta) \text { with } \\
\operatorname{slv}(f ; \alpha) \subset S \subset \operatorname{siv}(f ; \beta)\}, \\
\mathscr{F}_{\Delta, \mathbf{I}}^{+}=\{f \in C(X, \mathbf{I}): \text { whenever inf } f<\alpha<\beta<\sup f, \exists S \in \Sigma(\Delta) \text { with } \\
\operatorname{slv}(f ; \alpha) \subset S \subset \operatorname{slv}(f ; \beta)\},
\end{array}
$$

then we have $\mathscr{F}_{\Delta, \mathbf{R}}^{+}=\mathscr{F}_{\Delta, \mathbf{R}}^{++}$and $\mathscr{F}_{\Delta, \mathbf{I}}^{+}=\mathscr{F}_{\Delta, \mathbf{I}}^{++}$with respect to the uniformity $\mathscr{U}(C(X, \mathbf{R}))$. Thus, weak topology/uniformization results for hit-and-miss topologies are immediate consequences of our prior results. Proposition 3.2 and Theorem 3.3 become

3.5. Proposition. Let $X$ be a Hausdorff space, and let $\Delta$ be a Urysohn family of closed subsets of $X$. Let $A \in \mathrm{CL}(X)$, and let $\left\langle A_{\lambda}\right\rangle$ be a net in $\mathrm{CL}(X)$. The following are equivalent:

(1) $A=\tau_{\Delta}^{+}-\lim A_{\lambda}$

(2) $\forall f \in \mathscr{F}_{\Delta, \mathbf{R}}^{+}$we have $\lim _{\lambda} m_{f}\left(A_{\lambda}\right)=m_{f}(A)$;

(3) $\forall f \in \mathscr{F}_{\Delta, \mathrm{I}}^{+}$we have $\lim _{\lambda} m_{f}\left(A_{\dot{\lambda}}\right)=m_{f}(A)$.

3.6. Theorem. Let $X$ be a Hausdorff space, and let $\Delta$ be a family of closed subsets of $X$ containing the singletons. Then $\tau_{\Delta}^{+}$is uniformizable if and only if $\Delta$ is Urysohn.

In the statement of these results, there is no need to stipulate that $X$ be a uniform space, because hit-and-miss topologies are always admissible and the existence of a Urysohn family guarantees that the underlying space is completely regular. We list some special cases of interest. The nonempty closed subsets of a Hausdorff space $X$ are a Urysohn family if and only if $X$ is normal. In this case, with $\Delta=\mathrm{CL}(X)$, we have $\mathscr{F}_{\Delta, \mathbf{R}}^{+}=C(X, \mathbf{R})$. Thus, Proposition 3.5 yields the following result, observed in multifunction form by Choquet [Ch].

3.7. Corollary. Let $X$ be a normal space, and let $A$ and $\left\langle A_{\lambda}\right\rangle$ be nonempty closed subsets of $X$. Then $\left\langle A_{\lambda}\right\rangle$ is convergent to $A$ in the Vietoris topology if and only if for each $f \in C(X, \mathbf{R})$, we have $\lim _{\lambda} m_{f}\left(A_{\lambda}\right)=m_{f}(A)$.

When $\Delta=$ the nonempty compact subsets of a Hausdorff space $X$, then $\Delta$ is Urysohn if and only if $X$ is locally compact. Since the compact sets are closed under finite unions and arbitrary intersections, all functions in $\mathscr{F}_{\Delta, \mathbf{R}}^{+}$ have compact sublevel sets at height below $\sup \{f(x): x \in X\}$. We obtain the following result.

3.8. Corollary. Let $X$ be a locally compact Hausdorff space, and let $A$ and $\left\langle A_{\lambda}\right\rangle$ be nonempty closed subsets of $X$. Then $\left\langle A_{\lambda}\right\rangle$ is convergent to $A$ in the 
Fell topology if and only if for each $f \in C(X, \mathbf{R})$ such that $\operatorname{siv}(f ; \alpha)$ is compact for each $\alpha<\sup \{f(x): x \in X\}$ we have $\lim _{\lambda} m_{f}\left(A_{\lambda}\right)=m_{f}(A)$.

We cannot in general replace our distinguished class of functions in Corollary 3.8 by the family of continuous functions that have compact sublevel sets, for the existence of one such function forces sigma compactness on the space.

If we extend $\tau_{\Delta}^{+}$to $\operatorname{CL}(X) \cup\{\varnothing\}$ by taking as local base at $\varnothing$ all sets of the form $\left(S^{c}\right)^{+}$where $S \in \Sigma(\Delta)$, then we have the following translation of Corollary 3.4:

3.9. Corollary. Let $X$ be a Hausdorff space, and let $\Delta$ be a family of closed subsets of $X$ containing the singletons. Then the extended hit-and-miss topology $\tau_{\Delta}^{+}$on $\mathrm{CL}(X) \cup\{\varnothing\}$ is uniformizable if and only if $\Delta$ is Urysohn.

We now give a contribution of $\mathrm{R}$. McCoy characterizing second countability within the uniformizable hit-and-miss proximal hyperspaces.

3.10. Theorem. Let $\langle X, \mathscr{U}\rangle$ be a Hausdorff uniform space, and let $\Delta$ be a uniformly Urysohn family of closed subsets of $X$ containing the singletons. Then $\left\langle\mathrm{CL}(X), \tau_{\Delta}^{++}\right\rangle$is second countable if and only if $\Delta$ contains a countable subfamily $\Delta^{\prime}$ such that whenever $D \in \Delta$ and $A \in \mathrm{CL}(X)$ are far there exist $S \in \Sigma\left(\Delta^{\prime}\right)$ and $U \in \mathscr{U}$ such that $U[D] \subset S \subset A^{c}$.

Proof. First, suppose such a $\Delta^{\prime}$ exists. We show that $\tau_{\Delta}^{++}$is generated by a countable family of infimal value functions with values in $[0,1]$, which immediately gives second countability. Define $P \equiv\left\{\left(S_{1}, S_{2}\right) \in \Sigma\left(\Delta^{\prime}\right) \times \Sigma\left(\Delta^{\prime}\right)\right.$ : $U\left[S_{1}\right] \subset S_{2}$ for some $\left.U \in \mathscr{U}\right\}$. Let $p=\left(S_{1}, S_{2}\right) \in P$; since $S_{1}$ and $\operatorname{cl}\left(S_{2}^{c}\right)$ are far, the proof of Lemma 3.1 allows us to construct $f_{p} \in \mathscr{F}_{\Delta, \mathbf{I}}^{++}$ such that $f_{p}\left(S_{1}\right)=0$ and $f_{p}\left(\mathrm{cl}\left(S_{2}^{c}\right)\right)=1$. This defines a countable subfamily $\mathscr{F}^{\prime}=\left\{f_{p}: p \in P\right\}$ of $\mathscr{F}_{\Delta, \mathrm{I}}^{++}$. Now suppose $D \in \Delta$ and $A \in \mathrm{CL}(X)$ are far. By the definition of $\Delta^{\prime}$, there exist $S_{1}, S_{2}$ in $\Sigma\left(\Delta^{\prime}\right)$ and $U \in \mathscr{U}$ such that $U[D] \subset S_{1} \subset U\left[S_{1}\right] \subset S_{2} \subset A^{c}$. Choosing $f \in \mathscr{F}^{\prime}$ with $f\left(S_{1}\right)=0$ and $f\left(S_{2}^{c}\right)=1$, we get $f(D)=0$ and $f(A)=1$. Thus, $\mathscr{F}^{\prime}$ alone suffices to separate elements of $\Delta$ from far closed sets in the sense of Lemma 3.1(c), and the proof of Proposition 3.2 goes through with $\left\{m_{f}: f \in \mathscr{F}^{\prime}\right\}$ replacing $\left\{m_{f}: f \in \mathscr{F}_{\Delta, \mathbf{I}}^{++}\right\}$. Thus, the hyperspace is the weak topology determined by $\left\{m_{f}: f \in \mathscr{F}^{\prime}\right\}$.

Conversely, suppose the hyperspace is second countable. Then there exists a countable family $\left\{V_{i}: i \in \mathbf{Z}^{+}\right\}$of open subsets of $X$ and a countable subfamily $\Delta^{\prime}$ of $\Delta$ such that $\left\{V_{i}^{-}: i \in \mathbf{Z}^{+}\right\} \cup\left\{\left(D^{c}\right)^{+}: D \in \Delta^{\prime}\right\}$ is a subbase for $\tau_{\Delta}^{++}$. We show that $\Delta^{\prime}$ does the job. To this end, let $D \in \Delta$ and $A \in \operatorname{CL}(X)$ be far. By the uniformly Urysohn property, there is $S_{1} \in \Sigma(\Delta)$ and $U \in \mathscr{U}$ such that $U[D] \subset S_{1}$ and $U\left[S_{1}\right] \subset A^{c}$. Then $A \in\left(S_{1}^{c}\right)^{++}$, and so there exists $S_{2} \in \Sigma\left(\Delta^{\prime}\right)$ and open $V_{1}, V_{2}, \ldots, V_{n}$ such that

$$
A \in V_{1}^{-} \cap V_{2}^{-} \cap \cdots \cap V_{n}^{-} \cap\left(S_{2}^{c}\right)^{++} \subset\left(S_{1}^{c}\right)^{++} .
$$

Clearly, $S_{2} \subset A^{c}$. To show that $S_{1} \subset S_{2}$, suppose to the contrary that $x \in S_{1} \cap$ $S_{2}^{c}$. Choose for $i=1,2,3, \ldots, n, a_{i} \in A \cap V_{i}$. Then $\left\{x, a_{1}, a_{2}, \ldots, a_{n}\right\} \in$ $V_{1}^{-} \cap V_{2}^{-} \cap \cdots \cap V_{n}^{-} \cap\left(S_{2}^{c}\right)^{++}$whereas $\left\{x, a_{1}, a_{2}, \ldots, a_{n}\right\} \notin\left(S_{1}^{c}\right)^{++}$, a contradiction. Thus, $U[D] \subset S_{1} \subset S_{2} \subset A^{c}$, so that $\Delta^{\prime}$ has the desired property. 
By the Urysohn metrization theorem, second countability of $\tau_{\Delta}^{++}$when $\Delta$ is uniformly Urysohn gives metrizability of the hyperspace. Conversely, as $\mathrm{Di}$ Maio and Hola have recently observed [DMH], metrizability gives second countability, as the existence of a countable local base for $\tau_{\Delta}^{++}$at $X$ itself for metrizable $X$ gives separability of $X$; whence, the finite subsets of a fixed countable dense subset are dense in the hyperspace.

We close by stating the analog of Theorem 3.10 for hit-and-miss hyperspaces.

3.11. Theorem. Let $X$ be a Hausdorff space, and let $\Delta$ be a Urysohn family of closed subsets of $X$ containing the singletons. Then $\left\langle\mathrm{CL}(X), \tau_{\Delta}^{+}\right\rangle$is second countable if and only if $\Delta$ contains a countable subfamily $\Delta^{\prime}$ such that whenever $D \in \Delta$ and $A \in \mathrm{CL}(X)$ are disjoint there exists $S \in \Sigma\left(\Delta^{\prime}\right)$ with $D \subset$ int $S \subset$ $S \subset A^{c}$.

Again, metrizability is equivalent to second countability. Characterizations of second countability for both $\tau_{\Delta}^{++}$and $\tau_{\Delta}^{+}$without conditions on $\Delta$ are given in [DMH], assuming second countability of the underlying space $X$.

Most of the results of this paper for $\tau_{\Delta}^{++}$and $\tau_{\Delta}^{+}$were obtained under the assumption that $\Delta$ contained the singletons. This can be weakened to the following condition: for each $A \in \mathrm{CL}(X)$ and $x \in A^{c}$, there exists $D \in \Delta$ with $x \in D \subset A^{c}$. It is not hard to show that this condition characterizes the $T_{1}$ separation property for both hyperspaces (see, e.g., [Po1]), and it is also equivalent to the fact that neither hyperspace is properly enlarged upon replacing $\Delta$ by $\Delta \cup\{\{x\}: x \in X\}$.

\section{REFERENCES}

[At] H. Attouch, Variational convergence for functions and operators, Pitnam, New York, 1984.

[AB] $\mathrm{H}$. Attouch and $\mathrm{G}$. Beer, On the convergence of subdifferentials of convex functions, Sém. Anal. Convexe 21 (1991), exposé no. 8; Arch. Math. 60 (1993), 389-400.

[Be1] G. Beer, Support and distance functionals for convex sets, Numer. Funct. Anal. Optim. 10 (1989), 15-36.

[Be2] __ The slice topology: A viable alternative to Mosco convergence in nonreflexive spaces, Sém. Anal. Convexe 21 (1991), exposé no. 3; Nonlinear Anal. 19 (1992), 271-290.

[Be3] __ Lipschitz regularization and the convergence of convex functions, Numer. Funct. Anal. Optim. (to appear).

[BB] G. Beer and J. Borwein, Mosco and slice convergence of level sets and graphs of linear functionals, J. Math. Anal. Appl. 175 (1993), 53-67.

[BLLN] G. Beer, A. Lechicki, S. Levi, and S. Naimpally, Distance functionals and suprema of hyperspace topologies, Ann. Mat. Pura Appl. 162 (1992), 367-381.

[BL] G. Beer and R. Lucchetti, Weak topologies for the closed subsets of a metrizable space, Trans. Amer. Math. Soc. 335 (1993), 805-822.

[BT] G. Beer and R. Tamaki, On hit-and-miss hyperspace topologies, Comment. Math. Univ. Carolin. 34 (1993), 717-728.

[Ch] G. Choquet, Outils topologiques et métriques de l'analyse mathématique, Centre de documentation universitaire et S.E.D.E.S. réunis, Paris, 1966.

[DCNS] A. Di Concilio, S. Naimpally, and P. Sharma, Proximal hypertopologies, preprint.

[DMH] G. Di Maio and L. Hola, On hit-and-miss topologies, preprint.

[Ef] V. A. Efremovic, The geometry of proximity. I, Mat. Sb. 31 (1952), 189-200.

[Fe] J. Fell, A Hausdorff topology for the closed subsets of a locally compact non-Hausdorff space, Proc. Amer. Math. Soc. 13 (1962), 472-476. 
[FLL] S. Francaviglia, A. Lechicki, and S. Levi, Quasi-uniformization of hyperspaces and convergence of nets of semicontinuous multifunctions, J. Math. Anal. Appl. 112 (1985), 347-370.

[He] C. Hess, Contributions à l'ètude de la mesurabilité, de la loi de probabilité, et de la convergence des multifunctions, Thèse d'état, U.S.T.L. Montpellier, 1986.

[Jo] J. Joly, Une famille de topologies dur l'ensemble des fonctions convexes pour lesquelles la polarité est bicontinue, J. Math. Pures Appl. 52 (1973), 421-441.

[KT] E. Klein and A. Thompson, Theory of correspondences, Wiley, New York, 1984.

[Mi] E. Michael, Topologies on spaces of subsets, Trans. Amer. Math. Soc. 71 (1951), 152-182.

[NW] S. Naimpally and B. Warrack, Proximity spaces, Cambridge Univ. Press, Cambridge, 1970.

[Po1] H. Poppe, Eine Bemerkung über Trennungsaxiome im Raum der abgeschlossenen Teilmengen eines topologischen Raumes, Arch. Math. 16 (1965), 197-199.

[Po2] __ Einige Bemerkungen über den Raum der abgeschlossen Mengen, Fund. Math. 59 (1966), 159-169.

[SZ] Y. Sonntag and C. Zalinescu, Set convergences: An attempt of classification, Proc. Internat. Conf. on Differential Equations and Control Theory (Iasi, Romania, August 1990), revised version; Trans. Amer. Math. Soc. (to appear).

[Ve] W. Vervaat, Random upper semicontinuous functions and extremal processes, Centre for Mathematics and Computer Science report \#MS-R8801, Amsterdam, 1988.

[Wi] R. Wijsman, Convergence of sequences of convex sets, cones, and functions. II, Trans. Amer. Math. Soc. 123 (1966), 32-45.

Department of Mathematics, California State University, Los Angeles, Los Angeles, CALIFORNIA 90032 